\title{
Detection of Positron Implantation Profile in Different Materials
}

\author{
J. DRYZEK \\ Institute of Nuclear Physics, Polish Academy of Sciences \\ Radzikowskiego 152, 31-342 Kraków, Poland
}

The objective of this paper is experimental studies of the linear absorption coefficient, or mean penetration depth of positrons emitted from ${ }^{22} \mathrm{Na}$ isotope in different materials. For this purpose we constructed a new experimental setup which allows us to scan the depth implantation profile of positrons. For the studied metals: $\mathrm{Mg}, \mathrm{Al}$, the obtained values of mean penetration depth coincide well with those which can be calculated using the mathematical formula commonly used but for Si and $\mathrm{S}$ the discrepancy has been observed similarly like for polymers.

PACS numbers: 61.72.Ji, 78.70.Bj

\section{Introduction}

The knowledge of the distribution profile of implanted positrons is useful not only in experiments with isotope sources. For studies of layered, heterogeneous samples with nonuniform defect distribution it is well at least to be aware of the positron implantation range, because it can happen that these distributions coincide. The knowledge of positron implantation range is necessary for the positron emission tomography where the spatial distribution of a positron emitter is detected.

Basically, the implantation profile can be determined using the corrected value of the coefficients of positron mass absorption and backscattering. Nevertheless, the experiments and considerations of many authors do not lead to the common conclusions which could support this statement. Saoucha [1] suggests establishing two effective mass absorption coefficients pertaining, respectively, to the two regions below and above the certain critical thickness. This idea was reported earlier by Bisi et al. [2], who proposed to use two exponential functions with two different parameters of positron absorption in a mylar foil in the sandwiched 
geometry. Płotowski et al. [3] proposed rather another approach; the fraction of positrons passed throughout a foil must be averaged over the solid angle using only one value of the absorption coefficient.

Over decades several experimental techniques were used for detection of the positron implantation profile. The early experiments were based on the measurement of the intensity of a positron beam passed throughout thin foils [4]. The development of the more accurate detection techniques allows us to trace their distribution into the solid angle. For this purpose advantage is taken of the fact that the positron annihilation characteristics are different in different materials. The positron lifetime measurements have been used by Djourelov and Misheva [5] for determination of the fraction of positrons passed throughout the kapton foil of different thickness in a commonly used sandwich geometry. Linderoth et al. [6] in their experiment applied the measurements of the Doppler broadening of annihilation line. In this experiment the positron source enveloped in the nickel foil of different thickness were sandwiched between a number of different materials which backscattered positrons. One should emphasise the significant role of the backscattering phenomena in constitution of the positron implantation profile when the sandwich consists of different materials. For elimination of this phenomenon and still tracing the fate of implanted positrons into matter also the defects which can localised the thermalized positrons were applied [7]. Nevertheless in all these experiments the final distribution was deduced from the integrated number of positrons stopped or passed throughout a layer of a certain thickness.

The aim of this paper is the proposition of another type of experiment for the determination of the distribution of positrons implanted from the isotope source into the solid angle in a sandwiched geometry. The idea originated from those proposed by Brandt and Paulin [8]. They observed the sample illuminated by positrons using two scintillation detectors with $\mathrm{NaI}(\mathrm{Tl})$ crystals placed behind the long slits in lead shields. Moving the sample in the front of the long slits they were able to scan the depth distribution of implanted positrons. We intend to present the experimental setup and the measured values of the mean penetration depth in different materials. In our future works we intend to apply it for determination of the defect distribution in samples whose surface were damaged [9].

\section{Setup description}

In our experimental setup, whose scheme is depicted in Fig. 1, the main part is the HpGe coaxial detector, which is used for observation of either annihilation or $\gamma$ rays. This detector has the volume of $130 \mathrm{~cm}^{3}$ and the energy resolution (FWHM) of $1.38 \mathrm{keV}$ interpolated for the energy of $511 \mathrm{keV}$ and the peak to background ratio is about 130:1. The window of the detector was placed behind a $10 \mathrm{~cm}$ thick lead shield with the long slit of $100 \mu \mathrm{m}$ width. In the front of the slit the support was fixed which could precisely move in the direction perpendicular 
to the slit. The samples with the positron source were placed on the support. The micrometer screw, Fig. 1, controlled the position of the support. A stepping motor connected throughout the control unit to the PC computer initiated the rotation of the micrometer screw. The computer read in the sequenced mode the $\gamma$ ray spectrum via ORTEC TRUMP $2 \mathrm{k}$ card after fixing the position of the micrometer screw.

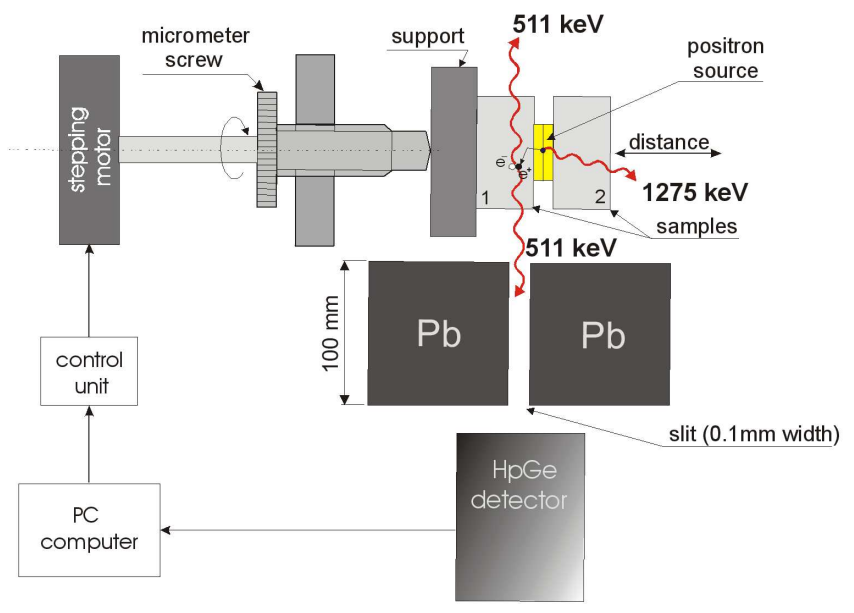

Fig. 1. The layout of the experimental setup used for the scanning of positron implantation profile.

As the positron source we used the conventional source based on the isotope ${ }^{22} \mathrm{Na}$ which was enveloped in the thin kapton foil of $7 \mu \mathrm{m}$ thick. The envelope has the shape of a circle of $10 \mathrm{~mm}$ in diameter and the activity of the source was $90 \mu \mathrm{Ci}$. Due to the glue on the edge used as an envelope seal the total thickness of the source located between the samples is usually higher than the thickness of the kapton foil. For our source it was $60 \mu \mathrm{m}$. It is worth noticing that in our setup we used a ten times lower activity of a positron source than that used by Brand and Paulin [8].

\section{The spatial resolution function}

Our positron source emits positrons and $\gamma$ rays of $1.275 \mathrm{MeV}$ which tags the positron emission. The $\gamma$ rays emission originates only in the region of the source or in the place where the isotope is distributed but the emission of annihilation quantum of $511 \mathrm{keV}$ marks the position where the positron finishes its life after implantation and thermarization. Thus moving the support, Fig. 1 and monitoring the total counts in the $1.275 \mathrm{MeV}$ line we can locate the position of the source placed between the samples and the same for the $511 \mathrm{keV}$ positron implantation profile. 
For testing of the setup we used two pieces of graphite plates $20 \mathrm{~mm} \times$ $30 \mathrm{~mm}$ and $4 \mathrm{~mm}$ thick as the samples. In Fig. 2a we present the integrated number of counts in the $1.275 \mathrm{MeV}$ line per second as a function of the distance (see Fig. 1) which is the position of the studied sandwich in the front of the slit. The obtained dependence can be described by a single Gaussian curve with the full width at the half maximum (FWHM) equal to $203 \mu \mathrm{m}$ and the central position at $x_{0}=905 \mu \mathrm{m}$. This curve could be treated as a spatial resolution function of our setup. Nevertheless, the width of the slit in the lead shield is equal to $100 \mu \mathrm{m}$ measured using a gap gauge and the slit between the samples with the source is equal to $60 \mu \mathrm{m}$. Thus we expected the FWHM close to $100 \mu \mathrm{m}$.

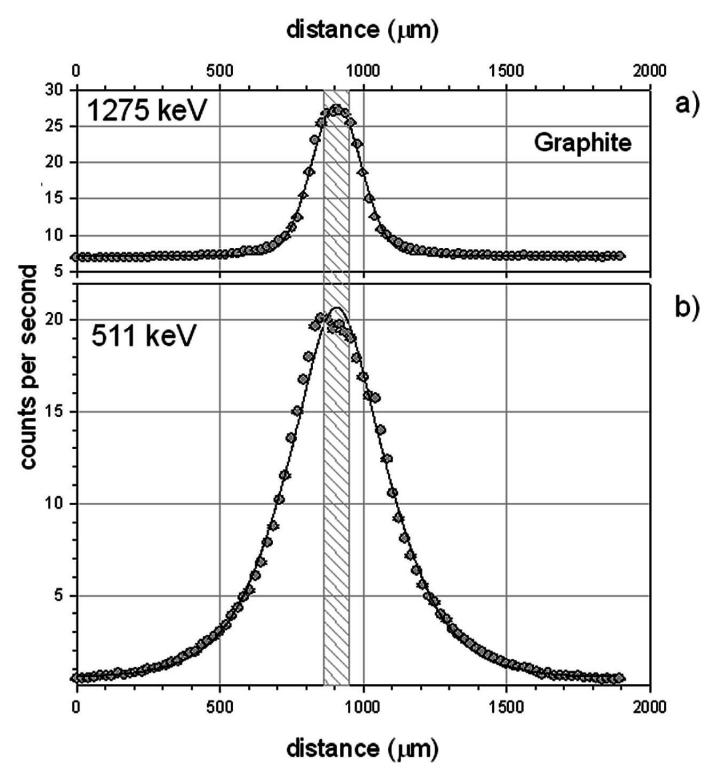

Fig. 2. The results of the measurements using the setup presented in Fig. 1. Figure (a) presents the integrated number of counts under the $\gamma$ line $1.275 \mathrm{keV}$ emitted from the source with the isotope ${ }^{22} \mathrm{Na}$ (closed circles) as a function of the position of the source sandwiched with the graphite samples. Figure (b) presents the integrated number of count under the line $511 \mathrm{keV}$ resulting from the positrons annihilation as a function of the sandwich position as well. The solid line represents the best fit of the relation (1) to the experimental points.

To support this we performed the Monte Carlo simulation of the operation of our setup. In the performed simulation we took into account the emission of the $\gamma$ rays of $1.2 \mathrm{MeV}$ from the source located in the front of the slit considering the absorption of the $\gamma$ quanta in the lead shield and their deflection due to the Compton scattering. The assumed absorption coefficient was equal to $666 \mathrm{~cm}^{-1}$ and the angle of deflection was deduced from the well-known relation for the energy 


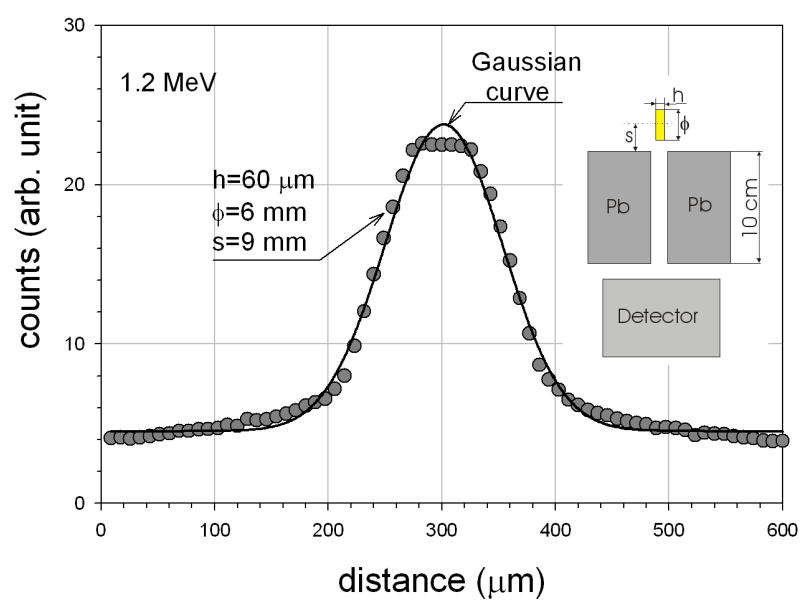

Fig. 3. The outcome of the Monte Carlo simulations of the setup presented in Fig. 1. The closed circles represent the results for the source of $60 \mu \mathrm{m}$ thick and $1 \mathrm{~cm}$ in diameter emitting $1.2 \mathrm{MeV} \gamma$ rays as it was in our experiment. The solid line represents the best fit of the Gaussian curve to the closed circles.

of the scattered $\gamma$ quanta. Due to the finite energy resolution of the detector the accepted energy of the $\gamma$ quanta was in the range from $1.19724 \mathrm{MeV}$ to $1.2 \mathrm{MeV}$. The first value arose from the energy resolution of our detector. In Fig. 3 the obtained results are depicted for the case when the source has a form of a cylinder of $6 \mathrm{~mm}$ in diameter and $60 \mu \mathrm{m}$ high, the distance of the middle of the source from the front of the lead shield is $9 \mathrm{~mm}$ like in our setup. First of all the obtained dependence can be described by the Gaussian curve but only in an approximate way. Note that either tails or top of the dependence exhibit a significant deviation from the best fit of the Gaussian curve. The FWHM of the Gaussian curve fitted to the simulated points equals to $122 \mu \mathrm{m}$ and this is 0.6 of the experimental value. We do not suppose that the additional, sequenced Compton scattering events, which were not taken into account in our simulation, could increase significantly the FWHM of the spatial distribution. We argue that geometrical effects could explain it, for instance when the source plane is not ideally parallel to the plane of the slit. The computer simulation has shown that when the plane of the source is slightly tilted regarding the axis parallel to the slit window, i.e. $1.5^{\circ}$ degree then the Gaussian FWHM increases to $214 \mu \mathrm{m}$. Due to the glue on the edge of the envelope with the source mentioned above, it is difficult to precisely control in the experiment whether the plane of the source is perfectly parallel to the slit plane. Nevertheless it is worth noticing that the shape of the experimental dependence from Fig. 2a, the flattened central part and risen tails, coincide well with the simulated one.

The broadening of the spatial resolution function almost by the factor of about two in comparison to the slit width was observed using another $\gamma$ ray source. 
Commonly, the ${ }^{22} \mathrm{Na}$ source can be simulated by the $\gamma$ ray source with the ${ }^{207} \mathrm{Bi}$ isotope which is the emitter of two $\gamma$ quanta of energy $569.7 \mathrm{keV}$ and $1063.7 \mathrm{keV}$. We used the source in the form of a cylinder of $1.5 \mathrm{~cm}$ in diameter and $110 \mu \mathrm{m}$ high with a much smaller activity $5 \mu \mathrm{Ci}$. We have found that the obtained results for the $569 \mathrm{keV}$ line can be described by the Gaussian curve with the FWHM = $209.7 \mu \mathrm{m}$.

One should mention that a similar discrepancy was observed by Brand and Paulin [8]. In their paper (see Fig. 4 in Ref. [8]) the resolution function was described by the Gaussian curve and the FWHM is about twice as high as the geometrical slit width, i.e. $100 \mu \mathrm{m}$.

Thus we can conclude that the spatial resolution function of our experimental setup can be described by the Gaussian curve with the FWHM ca. $209 \mu \mathrm{m}$. (The last value we adopted from the above measurement with the ${ }^{207} \mathrm{Bi}$ isotope.) The Gaussian curve is only the convenient approximation but searching for a better analytical function is required.

\section{The positron distribution}

In Fig. 2b we depicted the dependence of the integrated number of counts in the annihilation line per second which reflects the projection of the positron distribution in the sandwich on the plane perpendicular to the slit. As we expected, the dependence is much broader than that for the line of $1.275 \mathrm{MeV}$, Fig. 2a. For the description of the obtained distribution we followed the idea proposed by Brand and Pauli [8], Dryzek [9].

It was assumed that the implantation profile of emitted positrons fulfills the exponential law in the region of the samples but in the source region it is described by the Gaussian distribution (with the standard deviation denoted as $\sigma_{s}$ ) as it is with the distribution of $1.275 \mathrm{MeV}$ line presented above. Thus the measured number of counts $N$ per unit of time in the annihilation line as a function of position $x$ can be expressed as follows:

$$
\begin{aligned}
N(x) & =a d_{+} \int_{-\infty}^{\infty} \mathrm{d} \xi g(\xi-x) \\
& \times\left[(1-\varepsilon) \exp \left(-\left(\xi-x_{0}-d / 2\right) / d_{+}\right) \theta\left(\xi-x_{0}-d / 2\right)\right. \\
& \left.+\varepsilon \exp \left(-\left(-\xi+x_{0}-d / 2\right) / d_{+}\right) \theta\left(-\xi+x_{0}-d / 2\right)\right] \\
& +\frac{b}{\sqrt{2 \pi} \sigma_{s}} \exp \left(-\frac{\left(x_{0}-x\right)^{2}}{2 \sigma_{s}^{2}}\right)+b g
\end{aligned}
$$

where $d_{+}$is the mean penetration depth equal to the reciprocal value of the linear absorption coefficient for positrons, $a$ and $b$ are constant values, $b g$ represents the background, $d$ is the total source thickness and $x_{0}$ is the central position of the source. The function $g(x)$ is the spatial resolution function and $\theta$ is the 
Heaviside function defined as follows: $\theta(x)=1$ for $x \geq 0$ and $\theta(x)=0$ for $x<0$. To correct the slightly asymmetric position of the source we introduced the parameter $\varepsilon$, whose value should be close to 0.5 . In Fig. $2 \mathrm{~b}$ the solid line presents the best fit of Eq. (1) to the experimental points, the values of the fitted parameters were equal as follows: $d_{+}=132.2 \pm 3.9 \mu \mathrm{m}, d=106.9 \pm 0.4 \mu \mathrm{m}$, $b g=0.549 \pm 0.053, \varepsilon=0.506 \pm 0.005, a=7188 \pm 94, b=2067 \pm 84$. One should note that Eq. (1) was not able to describe the central part of the fitted dependence in a satisfactory way. This is due to the fact that in our source the isotope is distributed at a certain space and this spatial distribution is difficult to describe by an analytical function. Note the good accuracy of the obtained value of the mean penetration depth $d_{+}$, in this case it was about $3 \%$. We state that this accuracy is getting worse when the $d_{+}$value decreases. It is well visible for the experiment performed for pure well-annealed copper presented in Fig. 4. The positron distribution depicted in Fig. $4 \mathrm{~b}$ in this case almost coincides with the distribution of $1275 \mathrm{keV}$ line. The fitted value of the mean penetration depth equals to $10 \pm 60 \mu \mathrm{m}$. According to other experiments it is $25.9 \pm 4.0 \mu \mathrm{m}$ [7]. It

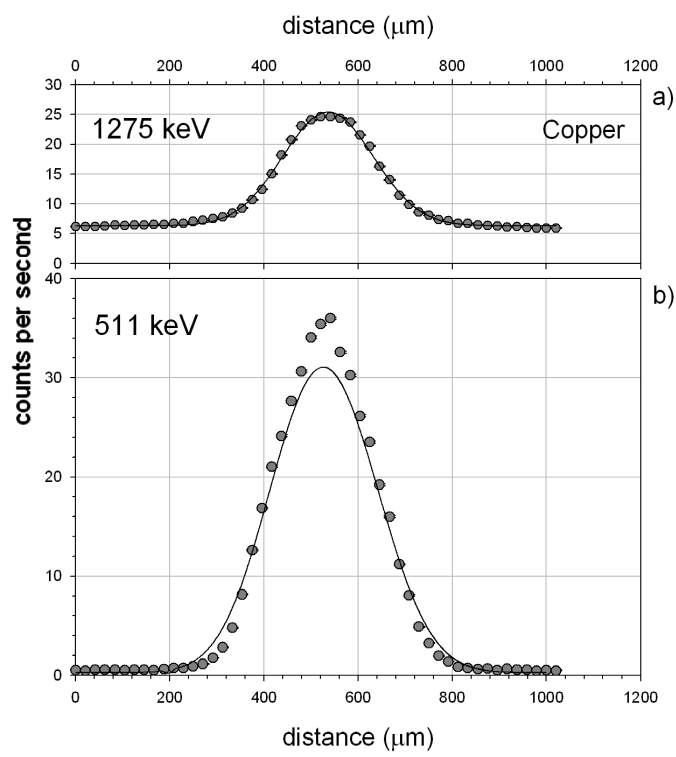

Fig. 4. The results of the measurements using the setup presented in Fig. 1. Figure (a) presents the integrated number of counts under the $\gamma$ line $1.275 \mathrm{keV}$ emitted from the source with the isotope ${ }^{22} \mathrm{Na}$ (closed circles) as a function of the position of the source sandwiched with the well-annealed copper samples. Figure (b) presents the integrated number of count under the line $511 \mathrm{keV}$ resulting from the positrons annihilation as a function of the sandwich position as well. The solid line represents the best fit of the relation (1) to the experimental points. 
indicates that our experimental setup can be used for determination of the positron penetration depth larger than $80 \mu \mathrm{m}$ when a reasonable accuracy is needed. To use it for metals where $d_{+}<50 \mu \mathrm{m}$ the setup should be improved. Instead of lead the tungsten shields should be used. This allows us to reduce the background by the factor of $c a$. two according to our simulations. The reduction of the thickness of the slit to $50 \mu \mathrm{m}$ allows us to improve the spatial resolution by the factor of about two as well. We believe that this helps to perform the studies of positron penetration depth in metals and their alloys as well.

In the Table we present the values of the measured mean penetration depth for positrons emitted from the ${ }^{22} \mathrm{Na}$ isotope. In the literature devoted to positron techniques it is frequently cited the empirical relation for this value:

$$
\frac{1}{d_{+}[\mathrm{cm}]}=17 \frac{\rho\left[\mathrm{g} \mathrm{cm}^{-3}\right]}{E_{\max }^{1.43}[\mathrm{MeV}]},
$$

where $\rho$ is the mass density of the solid and $E_{\max }$ is the maximum positron energy ( $0.54 \mathrm{MeV}$ for $\left.{ }^{22} \mathrm{Na}\right)$. Nevertheless, this relation proposed by Gleason et al. [4] was established for electrons more than fifty years ago. It is interesting to point out that for aluminium and magnesium the measured and calculated values of $d_{+}$using

TABLE

The values of the mean positron penetration depth for different materials of different densities obtained as a results of fitting the relation (1) to the experimental points measured using the presented setup.

\begin{tabular}{c|c|c}
\hline \hline Material & Density $\left(\mathrm{g} / \mathrm{cm}^{3}\right)$ & $\begin{array}{c}\text { Mean positron penetration } \\
\text { depth }(\mu \mathrm{m})\end{array}$ \\
\hline $\mathrm{Mg}$ & 1.74 & $132.1 \pm 1.8$ \\
$\mathrm{Al}$ & 2.68 & $93.4 \pm 2.3$ \\
$\mathrm{Si}$ & 2.33 & $72.3 \pm 2.8$ \\
$\mathrm{~S}$ & 2.07 & $110.2 \pm 4.6$ \\
Graphite & 2.26 & $132.2 \pm 3.9$ \\
Paraffin & 0.80 & $264.8 \pm 7.8$ \\
Kapton & 1.42 & $155.5 \pm 5.5$ \\
Teflon & 2.10 & $88.6 \pm 8.3$ \\
UHMWPE $^{a}$ & 0.92 & $237.6 \pm 12$ \\
EPDM $^{b}$ & 0.90 & $274.6 \pm 9.5$ \\
Plexiglass & 1.15 & $171.0 \pm 10$ \\
Glass & 2.38 & $65.6 \pm 5.0$ \\
Dry wood (birch) & 0.570 & $446.5 \pm 10$ \\
\hline
\end{tabular}

${ }^{a}$ ultra high molecular weight polyethylene,

${ }^{b}$ ethylene-propylene-diene rubber. 
this relation coincide well within error but for non-metals not exactly. For instance for sulfur the calculated value is $d_{+}=118 \mu \mathrm{m}$ and the measured one $d_{+}=110.2 \pm$ $4.6 \mu \mathrm{m}$. The significant discrepancy was observed for silicon, we got the value $d_{+}=72.3 \pm 0.28 \mu \mathrm{m}$ but according to (2) it should be close to $104.5 \mu \mathrm{m}$. In Fig. 5 we gathered the results from the Table presenting the dependence of $d_{+}$on the density. The solid line presents the empirical relation (2). It is well visible that the positron implantation range depends not only on the density of implanted material.

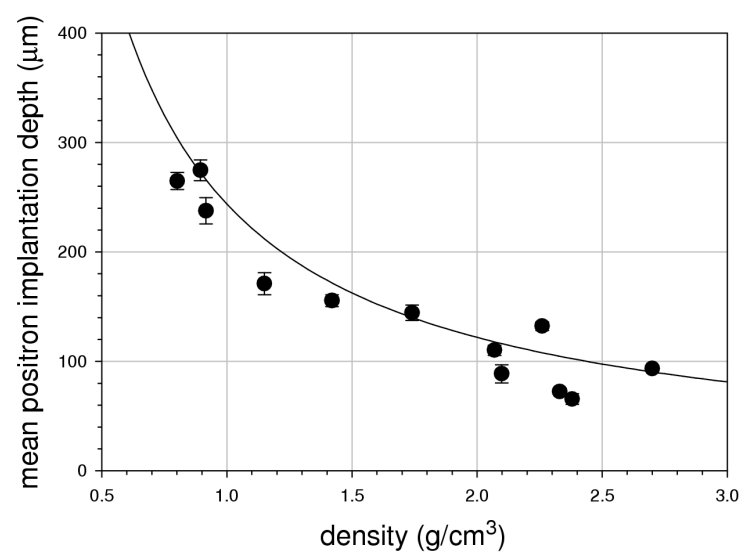

Fig. 5. The dependence of the mean penetration depth as a function of the density of the measured materials taken from the Table. The solid line represents the relation (2) obtained by Gleason et al. [4].

Mourino et al. [10] proposed to include into the empirical relation also the atomic number of implanted material. One should note that the proposed description of our experimental data is only a crude approximation. The transport of energetic electrons or charged particles implanted from the radioactive source into a medium has been attacked by many authors using different methods, because generally it is a complex problem. The most appropriate is to use the transport equations like, Boltzmann transport equation [11], Fokker-Planck-diffusion equation [12], the Lewis equation $[13,14]$ or Monte Carlo method [15] and recently the application of the Fermi age equation [16]. Nevertheless, the descriptions are very complicated, they require many parameterizations and do not lead to the simple conclusions. We think that future experimental studies of positron implantation process using the presented setup allow us to learn more about the slowing down process and find a reliable description.

\section{Conclusion}

The presented setup enabled us to scan the projection of the positron implantation profile. Despite still low spatial resolution of about $200 \mu \mathrm{m}$ the details 
of this profile have been observed in less dense metals ( $\mathrm{Al}$ and $\mathrm{Mg}$ ), non-metals, and in polymers. The positron mean implantation depths were determined for these materials using the approximate relation with the satisfactory accuracy, less than $4 \%$. Future improvements in the design of the setup should give possibilities to extend the scope of the studied materials and to find more details in the implantation profile.

\section{References}

[1] A. Saoucha, J. Appl. Phys. 85, 1802 (1999).

[2] A. Bisi, G. Gambarini, L. Zappa, Nuovo Cimento B 53, 426 (1979).

[3] K. Płotowski, T.J. Panek, J. Kansy, Nuovo Cimento D 10, 933 (1988).

[4] G.I. Gleason, J.D. Taylor, D.L. Tabern, Nucleonics 8, 12 (1951).

[5] N. Djourelov, M. Misheva, J. Phys. 8, 2081 (1996).

[6] S. Linderoth, H.E. Hansen, B. Nielsen, K. Petersen, Appl. Phys. A 33, 25 (1984).

[7] J. Dryzek, E. Dryzek, Phys. Status Solidi A 179, 337 (2000).

[8] W. Brandt, R. Paulin, Phys. Rev. B 15, 2511 (1977).

[9] J. Dryzek, to be published in Appl. Phys.

[10] M. Mourino, H. Läbl, R. Paulin, Phys. Lett. A 71, 106 (1979).

[11] T.M. Tran, P.-A. Haldy, J. Ligou, T. Lefvert, Atomkernenergie Kerntechnik 36, 218 (1980).

[12] H.A. Bethe, M.E. Rose, L.P. Smith, Proc. Am. Philos. Soc. 78, 573 (1938).

[13] H.W. Lewis, Phys. Rev. 78, 526 (1950).

[14] L.V. Spencer, Phys. Rev. 98, 1597 (1955).

[15] W. Williamson, Jr., G.C. Duncan, Am. J. Phys. 54, 262 (1986).

[16] D.T. Britton, G.A. Wigger, A. Seeger, Nucl. Instrum. Methods Phys. Res. B 164-165, 137 (2000). 